\title{
Clinical characteristics and CT features of hepatic epithelioid haemangioendothelioma and comparison with those of liver metastases
}

\author{
Xiaopeng Wang, Pan Liang, Peijie Lv, Rui Li, Ping Hou and Jianbo Gao*
}

\begin{abstract}
Background: To analyse clinical characteristics and computer tomography (CT) findings of hepatic epithelioid haemangioendothelioma (HEH) and to determine differential features compared with liver metastasis (LM).

Methods: This retrospective study included 80 patients with histopathologically confirmed HEH $(n=20)$ and LM ( $n=60)$ of different primary tumours who underwent dynamic contrast-enhanced CT scans. CT findings included the location, contour, size, number, margin, and density of lesions, the patterns and degree of contrast enhancement of lesions, vascular invasion and changes in other organs. The enhancement ratio (ER) and tumour-to-normal parenchyma ratio (TNR) were calculated. Receiver operating characteristic curves (ROCs) were used to determine areas under the curve (AUCs).

Results: About 65\% of HEH lesions were located in submarginal areas. Significant differences were observed between HEH and LM patients in age, sex, and tumour marker positivity $(p<0.05)$. HEH showed minimal to slight enhancement, thin ring-like enhancement in arterial phase, and slight, homogeneous, progressive enhancement in the portal phase. HEH presented capsule retraction, and the "target" sign and the "lollipop" sign were significantly more frequent than in LM $(p<0.05)$. The ER and TNR in the arterial phase of HEH were lower than those of LM $(p<0.05)$. AUCS of ER and TNR in the arterial phase were 0.74 and 0.73 , respectively.
\end{abstract}

Conclusion: Lesions in subcapsular locations, capsular retraction, slight and thin ring-like enhancement, "target" and "Iollipop" signs and lower ER and TNR in the arterial phase may represent important features of HEH compared with LM.

Keywords: X-ray computed tomography, Hepatic epithelioid haemangioendothelioma, Liver metastases

\section{Keypoints}

- Dynamic CT imaging reveals typical features of $\mathrm{HEH}$, including subcapsular location and nodule coalescence, capsular retraction, intralesional calcifications, and ring-like enhancement.

- When the typical "target" sign and "lollipop" sign are present, HEH should be highly suspected.

*Correspondence: gaojianbovip18@163.com

Department of Radiology, The First Affiliated Hospital of Zhengzhou

University, No. 1, East Jianshe Road, Zhengzhou 450052, Henan Province, China
- When combined with clinical data, a lower AER and TNR, and CT imaging can help to improve differential diagnosis of $\mathrm{HEH}$.

\section{Background}

Epithelioid haemangioendothelioma usually arises in soft tissue and rarely occurs in the liver. Hepatic epithelioid haemangioendothelioma $(\mathrm{HEH})$ is a rare primary vascular tumour with low to intermediate malignancy [1]. $\mathrm{HEH}$, with a prevalence of 1 per 1,000,000 individuals [2], was first described by Weiss and Enzinger in 1982 [3]. In 
1984, Ishak et al. [4] reported a series of HEHs and collected 32 cases from the literature. HEH is predominant in young and middle-aged women, though aetiologic factors remain unclear. Moreover, clinical and laboratory examinations of $\mathrm{HEH}$ are frequently non-specific. Given the variable clinical features and absence of useful diagnostic serum tumour markers, approximately $60 \%$ to $80 \%$ of HEHs are initially misdiagnosed [5]. Nevertheless, long-term survival in $\mathrm{HEH}$ is possible after successful liver resection or transplantation, even with the appearance of extrahepatic involvement [6]. Hence, it is important to diagnose $\mathrm{HEH}$ early and differentiate it from other malignant hepatic tumours before it multiple lesions develop. Currently, imaging plays an important role in diagnosis. There are many reports on the imaging characteristics of $\mathrm{HEH}$, including those obtained via ultrasound (US), computer tomography (CT), magnetic resonance imaging (MRI) and positron emission tomography/computer tomography (PET/CT) [7-10]. Most HEHs show multiple lesions and minimal to slight and king-like enhancement, and these features are similar to those of liver metastases (LMs); therefore, HEH is often misdiagnosed as LM. Because LM is more invasive than $\mathrm{HEH}$, with poor prognosis and different treatment strategies, understanding its imaging features will help to reduce difficulty in differential diagnoses in the clinic. To our knowledge, no studies have reported imaging signs for differentiating HEH from LM through indepth quantitative data-based assessment. Because of its usefulness in a variety of situations and ready availability, CT has been recommended as part of the initial workup for many tumours and subsequent surveillance for metastatic disease after a diagnosis of primary cancer during the same examination [11]. Thus, the purpose of this study was to investigate clinical characteristics and $\mathrm{CT}$ features of HEH and assess their value in differentiating HEH from LM. In general, comparison of follow-up images has powerful value for selecting HEH treatment plans and evaluating prognosis.

\section{Methods}

\section{Patients}

We retrospectively collected 24 patients with HEH and 1068 patients with LM from different primary tumours, as confirmed by pathology, between January 2014 and December 2020. All clinical and imaging data were obtained from the hospital information system (HIS) and picture archiving and communication system (PACS). The inclusion criteria were as follows: (1) patients who underwent biopsy or surgical resection for pathological results such as "HEH"; (2) histopathological proof of at least one LM, and the primary disease of LM was also confirmed by pathology; and (3) patients who underwent a dynamic contrast-enhanced CT scan within 20 days prior to surgery or biopsy. The exclusion criteria were as follows: (1) pathology slides inadequate or unavailable for review; (2) patients who had incomplete or inadequate CT images, images of insufficient image quality, missing medical charts; (3) patients who had received treatment (including local therapy, systemic chemotherapy, or hepatectomy) prior to the CT scan; and (4) coexistence with other malignancies or patients with marked hepatic steatosis. A total of 276 patients $(\mathrm{HEH}=20, \mathrm{LM}=256)$ met the criteria; however, there was a larger difference in number between the groups. Of the 256 patients with pathologically proven LM, 60 were selected, resulting in a matched control group at a ratio of 3:1 to the patients with HEH. The groups were matched in terms of year distribution, age range and classification of intrahepatic lesions. Ultimately, 80 patients $(\mathrm{HEH}=20, \mathrm{LM}=60)$ comprised our study population (Fig. 1). The underlying primary extrahepatic malignancy of LM included colorectal cancer $(n=21)$, gastric cancer $(n=13)$, pancreatic cancer $(n=10)$, breast cancer $(n=5)$, lung cancer $(n=4)$, adenocarcinoma of the duodenum $(n=2)$, extrahepatic bile duct cancer $(n=2)$, ampulla of Vater cancer $(n=1)$, gallbladder cancer $(n=1)$, and renal cell carcinoma $(n=1)$.

\section{Scan protocol}

Non-enhanced and dual-phase dynamic contrastenhanced CT scans of the abdomen were performed for all patients using a 16-channel multi-detector CT scanner (Brilliance 16, Philips Medical Systems, Cleveland, $\mathrm{OH}, \mathrm{USA}$ ) or a 64-channel multi-detector CT scanner (Discovery CT750 HD CT Scanner, GE Healthcare Milwaukee, WI, USA). The parameters of the Brilliance 16 scanner were as follows: detector collimation, $1.5 \mathrm{~mm}$; pitch, 1.25:1; tube voltage, $120 \mathrm{kVp}$; tube current, 80-270 mAs; rotation time, $0.6 \mathrm{~s}$. The parameters of the Discovery CT750 scanner were as follows: detector collimation, $0.625 \mathrm{~mm}$; pitch, 1.375:1; tube voltage, $120 \mathrm{kVp}$; tube current, 80-270 mAs; rotation time, $0.5 \mathrm{~s}$. Dynamic contrast enhancement was performed by intravenous administration of $1.5 \mathrm{~mL} / \mathrm{kg}$ iodinated contrast agent $(350 \mathrm{mg} \mathrm{I} / \mathrm{mL})$ at a rate of $3.5 \mathrm{~mL} / \mathrm{s}$ using an automatic bolus-tracking technique. After the injection, arterial phase scans were started $10 \mathrm{~s}$ after the attenuation threshold of the descending thoracic aorta reached 100 Hounsfield units. Portal phase scanning was performed $30 \mathrm{~s}$ after the arterial phase.

\section{Clinical analysis, treatment and follow-up}

A clinical attending physician with 5 years of experience retrospectively reviewed the clinical data, including age, sex, chief complaint, and laboratory examination. Chief 
Patients who had pathologically proven $\mathrm{HEH}(\mathrm{n}=24)$ or LM ( $\mathrm{n}=1068)$ between January 2014 and December 2020

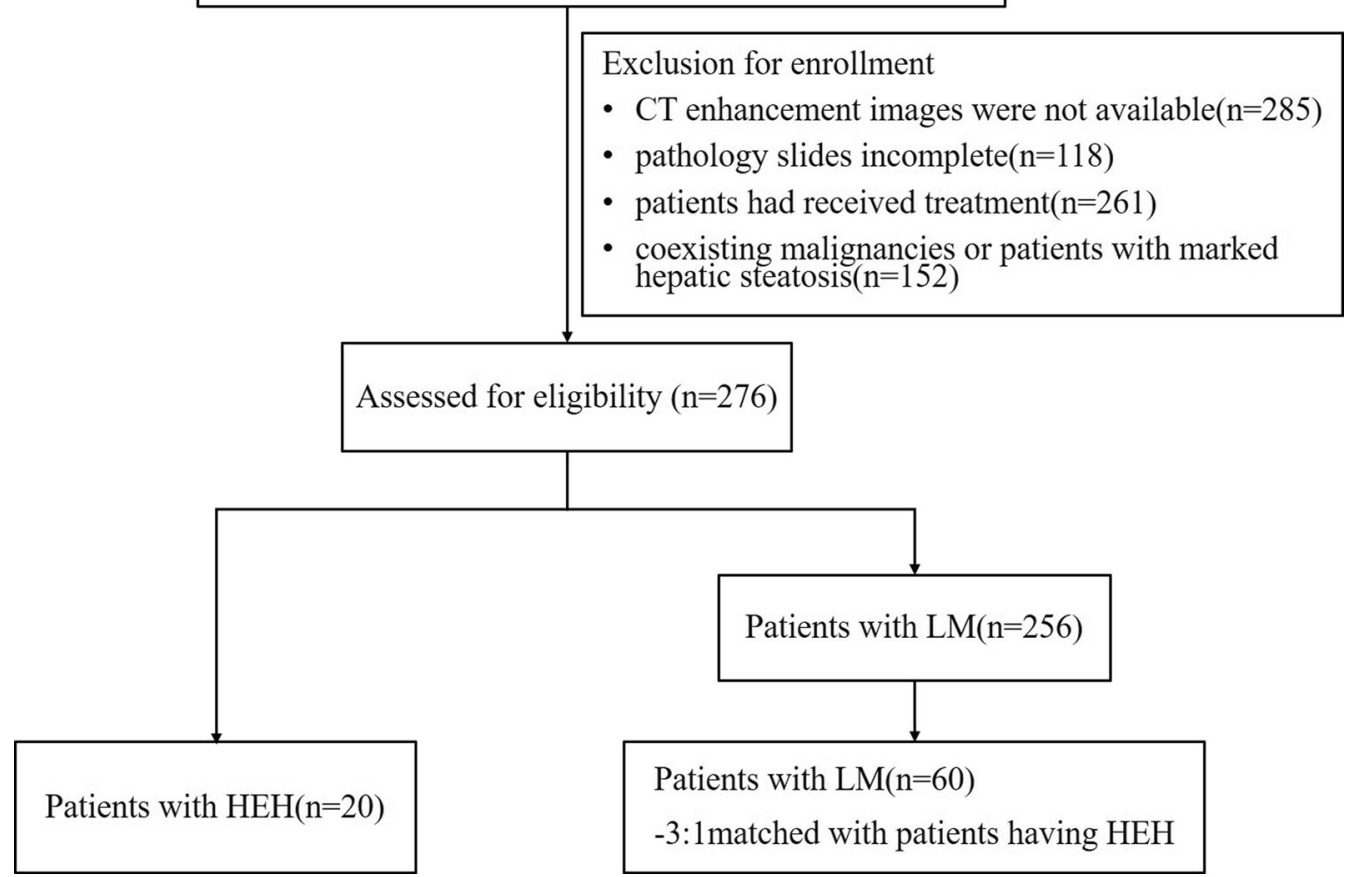

Fig. 1 A summary flowchart of the study population. HEH hepatic epithelioid haemangioendotheliomas, LM liver metastasis

complaints were classified as abdominal discomfort or pain, fever, weak, weight loss, poor appetite, melena, haematemesis, asymptomatic and other symptoms. Laboratory examinations were as follows: liver function, tumour markers, and tumour abnormal protein (TAP). Clinical treatment and follow-up results were recorded for the patients.

\section{Image analysis}

All CT images were analysed by two imaging diagnosticians (with 10 and 12 years of abdominal imaging experience) independently using a PACS. Although the readers were aware of the alternative diagnoses of HEH or LM, they were blinded to whether each lesion belonged to the HEH or LM group and to the clinical information and histopathological results. After independent image analysis, interobserver agreement was assessed for the evaluated CT imaging parameters; disagreements were resolved by consensus after reassessment of the images by the readers together. All images were analysed with regard to the following: (1) basic aspects-the maximum diameter (on axial images), number and classification (solitary nodular type, multifocal nodular type, and diffuse type), contour (round, round-like or strike-like, regular or irregular), margin (ill- or well-defined), location (scattered distribution, liver subcapsular) and density of the lesion (homogeneous or heterogeneous, hypo-, iso- or hyperdense, relative to the adjacent normal liver parenchyma) and the presence of a coalescence of lesions in cases of multiple lesions; (2) special signs and enhancement features [10]-(1) subcapsular location (any portion of the lesion in contact with the liver capsule); (2) capsular retraction (indentation of the hepatic contour for lesions with a subcapsular location); (3) rimlike (thin or thick peripheral enhancement encircling the lesion); (4) peripheral nodular enhancement (enhanced peripheral nodular areas); (5) "target" sign [12] (minimally enhanced centre and an enhanced inner peripheral rim juxtaposed with a minimally enhanced outer rim); (6) "lollipop" sign [12] (compression displacement or truncation of adjacent vessels around the lesion in portal venous phase); (7) calcification (lesions showing point, nodular or irregular high density, similar to that of bone); and (8) abnormal perfusion in the arterial phase (hepatic parenchyma around lesions showing patchy enhancement in the arterial phase and disappearance in the portal venous phase). When evaluating the outline and size of intrahepatic lesions, the observer should fix the abdominal window (window width $240 \mathrm{HU}$, window level $40 \mathrm{HU}$ ), whereas the location, shape, enhancement feature and other signs of the lesions can be appropriately adjusted according to the situation. 
Quantitative analysis was performed by measuring the attenuation of the lesion, and the normal parenchyma (Hounsfield units, HU) in the arterial and portal phases was measured in a circular or irregular region of interest (ROI). First, a slice showing the maximum diameter of the lesion on the axial image was selected. The ROI was then manually drawn within the boundary of the tumour, attempting to contain the whole tumour as much as possible. For all measurements, the size and shape of the ROI were kept consistent between the two phases, and the information from three lesion images of multiple lesions (where the lesion diameter was between 2 and $5 \mathrm{~cm}$ ) was obtained to calculate the average value. The means were then used to calculate the arterial enhancement ratio (AER) of the tumour (HU arterial-HU plain/HU plain), portal enhancement ratio (PER) (HU portal-HU plain/HU plain) [13], arterial tumour to normal parenchyma ratio (ATNR) (HU arterial/HU liver), and portal tumour to normal parenchyma ratio (PTNR) (HU portal/HU liver) [14]. The enhancement degree during contrast-enhanced imaging can be divided into minimal enhancement (CT attenuation changes less than $10 \mathrm{HU}$ ), slight enhancement (CT attenuation changes between 10 and $20 \mathrm{HU}$ ), or progressive moderate enhancement (CT attenuation changes more than $20 \mathrm{HU}$ ) [15].

\section{Histopathologic examination}

All HEH cases were determined by histopathology, including needle biopsy under ultrasound $(n=2)$ or CT $(n=14)$ guidance and surgical resection $(n=4)$. Each case of $\mathrm{HEH}$ was diagnosed based on light microscopic examinations, including haematoxylin-eosin and immunohistochemical staining results, confirming the endothelial origin of the tumour cells according to the tumour classification of the World Health Organization [16]. Microscopically, the tumours consisted of proliferated fibrous tissue intermingled with abundant vacuolated cytoplasm epithelioid cells. Endothelial markers (mainly factor VIII-related antigen, CD34, CD31, Ki-67 $5 \%)$ were positive according to immunohistochemical staining. All LMs were confirmed by pathology, clinical medical history and CT/MRI imaging during follow-up.

\section{Statistical analysis}

All statistical calculations were performed using Statistical Package for the Social Sciences (SPSS 20.0, Chicago, IL, USA). Categorical variables, including clinical characteristics (e.g., sex, main symptoms, laboratory examination) and qualitative CT features (e.g., classification, location, contour, border, density, enhancement pattern and degree, special signs, extrahepatic involvement), are described as frequencies or percentages. The $x^{2}$ test (Pearson and continuity correction) and Fisher's exact test were employed to evaluate differences between the groups. Continuous variables (age, ER and TNR) that followed a normal distribution are reported as the means and standard deviation and were compared using Student's $t$-test (including the corrected $t$-test). Receiver operating characteristic (ROC) curves of ER and TNR were obtained to generate the area under the curve (AUC) and an optimal cut-off, at which the sum of the sensitivity and the specificity was maximum. In general, an AUC between 0.5 and 0.7 suggests low diagnostic value, between 0.7 and 0.9 suggests medium diagnostic value, and between 0.9 and 1 suggests high diagnostic value. Sensitivity, specificity, and odds ratios (ORs) with 95\% confidence intervals (CIs) were analysed for variables differing significantly between the two groups. Kappa statistics were applied to determine the interobserver agreement of each variable. The Spearman correlation test was used to analyse correlation among $\mathrm{CT}$ features, treatment and clinical results. A $\kappa$ value of up to 0.20 was interpreted as slight agreement, $0.20-040$ as fair agreement, $0.40-0.60$ as moderate agreement, $0.60-0.80$ as substantial agreement, and $>0.80$ as almost perfect agreement. Statistical significance was defined by a $p$ value $<0.05$.

\section{Results}

\section{Clinical information, treatment and follow-up}

Table 1 summarises the clinical characteristics, treatments and follow-up results of the HEH patients (5 males and 15 females; median age, 41 and 42 years; range, 21-71). The basis for decision-making regarding treatment for $\mathrm{HEH}$ was the histopathologic classification, and the mode of hepatic involvement and the presence or absence of extrahepatic involvement were the main considerations. In general, the rate of progression, severity of signs and symptoms, and response to treatment methods should be considered for each patient. Treatment details were not available for 2 patients. The remaining 18 patients were managed with no treatment $(3 / 20,15 \%)$, liver resection $(4 / 20,20 \%)$, transcatheter arterial chemoembolisation (TACE) $(2 / 20,10 \%)$ and radiofrequency ablation (RFA) $(1 / 20,5 \%)$, chemotherapy $(2 / 20,10 \%)$, and complex therapy (chemotherapy, radiotherapy and immunotherapy) $(6 / 20,30 \%)$. During the follow-up, serial clinical and imaging information were available for 16 patients, which allowed evaluation of tumour progression. Of the remaining four patients, only telephone follow-up data were available for one, with no relevant imaging information, and the remaining 3 


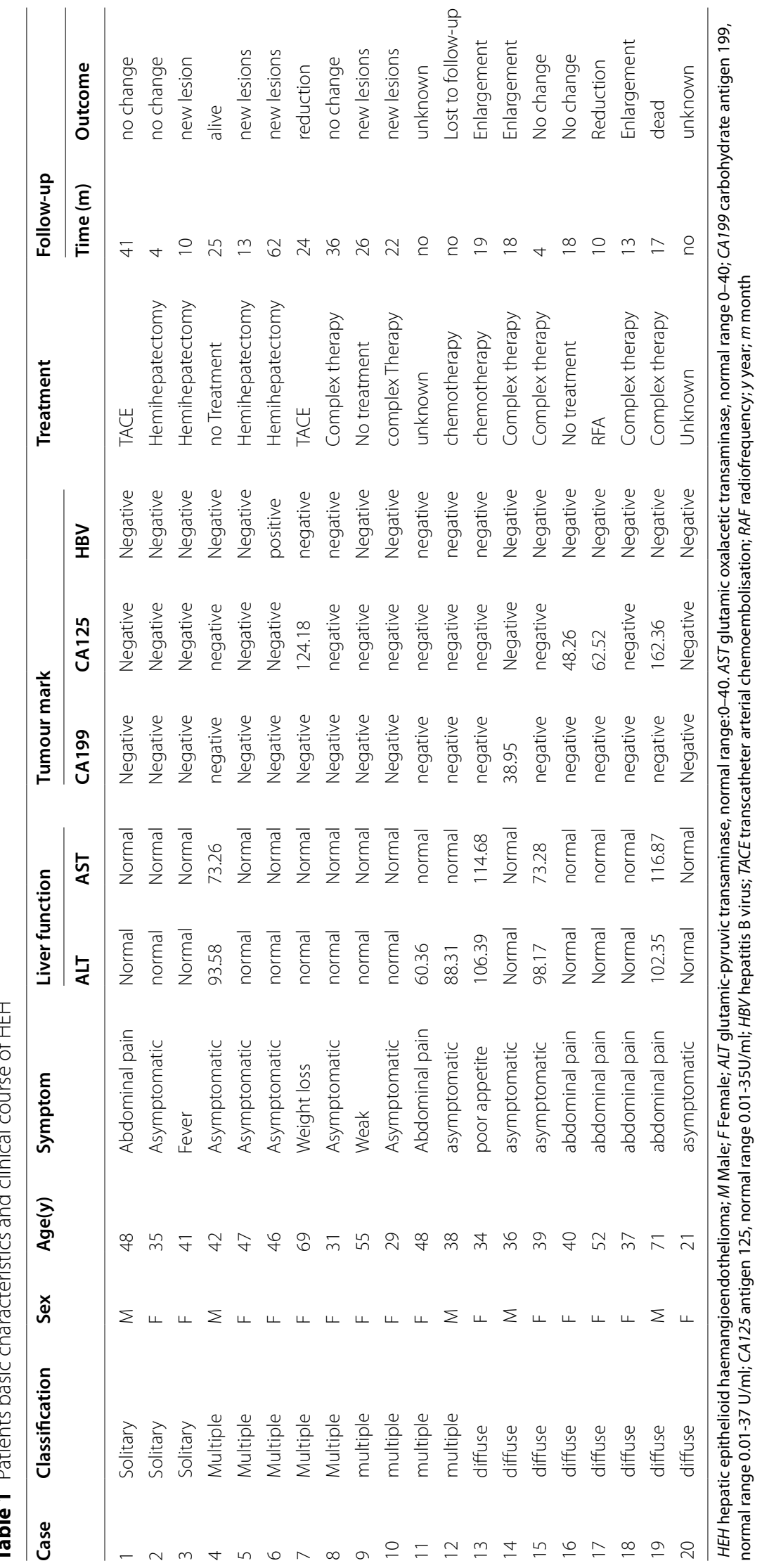


patients were lost to follow-up. At the last follow-up (4-62 months), 15 patients were alive; 1 patient had died due to underlying disease (digestive and respiratory diseases). Reduction, no obvious change, enlargement and new lesions were found in two, five, three and five patients, respectively, on the final CT images compared to the original CT images.

\section{Imaging findings of $\mathrm{HEH}$}

All CT imaging findings and enhancement patterns of the HEH patients are summarised in Table 2.

(1) The solitary nodular type was detected in 3 patients (3/20, 15\%), including 3 solitary lesions, all of which were located in the right lobe of the liver. Only 1 patient showed heterogeneous density with spot calcification; the other patients showed homogeneous density. One patient exhibited peripheral nodular enhancement, with enlarged feeding arteries and hyper-perfusion in the arterial phase. A "target" sign and portal vein branch invasion were detected in one patient (Fig. 2).

(2) The multifocal nodular type was detected in 9 patients $(9 / 20,45 \%)$. The lesions were located in the right lobe of the liver in only one patient; the whole liver was involved in all of the remaining 8 patients. Coalescent lesions were detected in 6 patients $(6 / 9$, $66.7 \%$ ). One patient showed multiple nodules with calcifications; the other 8 patients showed hypoattenuation compared with the normal liver parenchyma on non-contrast CT imaging. More than $80 \%$ of the lesions were peripheral and extended to the liver margin. Retraction of the liver capsule overlying the tumour nodules was detected in 10 lesions of 6 patients. Six patients showed minimal and slight enhancement in the arterial phase; 5 patients showed thin ring-like enhancement. Four patients exhibited slight to moderate progressive centripetal enhancement in the portal phase. In 5 patients, "target" sign enhancement in the portal phase was detected. The "lollipop" sign was observed in 8 lesions of 4 patients (Fig. 3).

(3) The diffuse type was observed in 8 patients $(8 / 20$, 40\%). CT features included diffuse low-density lesions with minimal residual areas of normal liver parenchyma, multiple nodules and confluent masses with strip-like enhancement located at the edge of the liver. Coalescent lesions were detected in most of the cases $(7 / 8,87.5 \%)$. Changes similar to those in liver cirrhosis were detected in 2 patients, such as liver morphological changes, proportion imbalance, and widened hepatic hilar and hepatic fissures (Fig. 4). Multiple lesions displayed slight or ring-like enhancement in the arterial phase and gradual homogeneous or heterogeneous enhancement in the portal phase. One patient showed involvement of lymph nodes; both the peritoneum and lung were invaded in another patient (Fig. 5). Stenosis or occlusion of the branches of the portal and hepatic veins was also detected in 2 patients (Fig. 6).

\section{Correlation between $\mathrm{CT}$ features, therapeutic decisions and clinical outcomes of $\mathrm{HEH}$}

The classification, location, distribution, contour, border, density, enhancement pattern and special signs of the lesions did not correlate with therapeutic decisions or clinical outcomes. Lesion size was modestly associated with the therapeutic decision (correlation coefficient $k=0.566, p=0.009$ ). In addition, there was a certain correlation between the "target sign" and clinical outcome (correlation coefficient $k=0.504, p=0.024$ ).

\section{Comprehensive comparative analysis of $\mathrm{HEH}$ and LM}

Table 3 summarises the comparison of clinical characteristics between HEH and LM. There were significant differences between the two groups in age, sex, main symptoms, and laboratory examination $(p<0.05)$. The majority of $\mathrm{HEH}$ patients were middle-aged women, most of whom had no obvious symptoms (50\%), normal liver function (70\%) and normal tumour markers (70\%); the majority of LM patients were middle-aged and elderly, with no sex predilection, who had abnormal liver function (57\%) and increased tumour markers (47\%).

Table 4 summarises the comparison of quantitative and qualitative CT findings between HEH and LM. A significant difference was observed in the distribution of tumour location $(p<0.000)$. Most of the HEH masses $(13 / 20,65 \%)$ were located in the subcapsular region of the liver, whereas LM had no obvious distribution characteristics. With regard to the lesion border, 13 (65\%) of the $20 \mathrm{HEH}$ masses were well defined, and 42 (70\%) of the 60 LM were ill defined. Minimal to slight, thin ring-like enhancement in the arterial phase and slight enhancement in the portal phase were observed in most of the HEH masses; slight to moderate, thick ring-like enhancement in the arterial phase and moderate enhancement in the portal phase were observed in most LMs. In HEH, the frequencies of capsular retraction, the "target" sign, and the "lollipop" sign were significantly higher than those in LM $(p<0.05)$. The presence of extrahepatic involvement, including lymph node, peritonea and bone invasion, was 


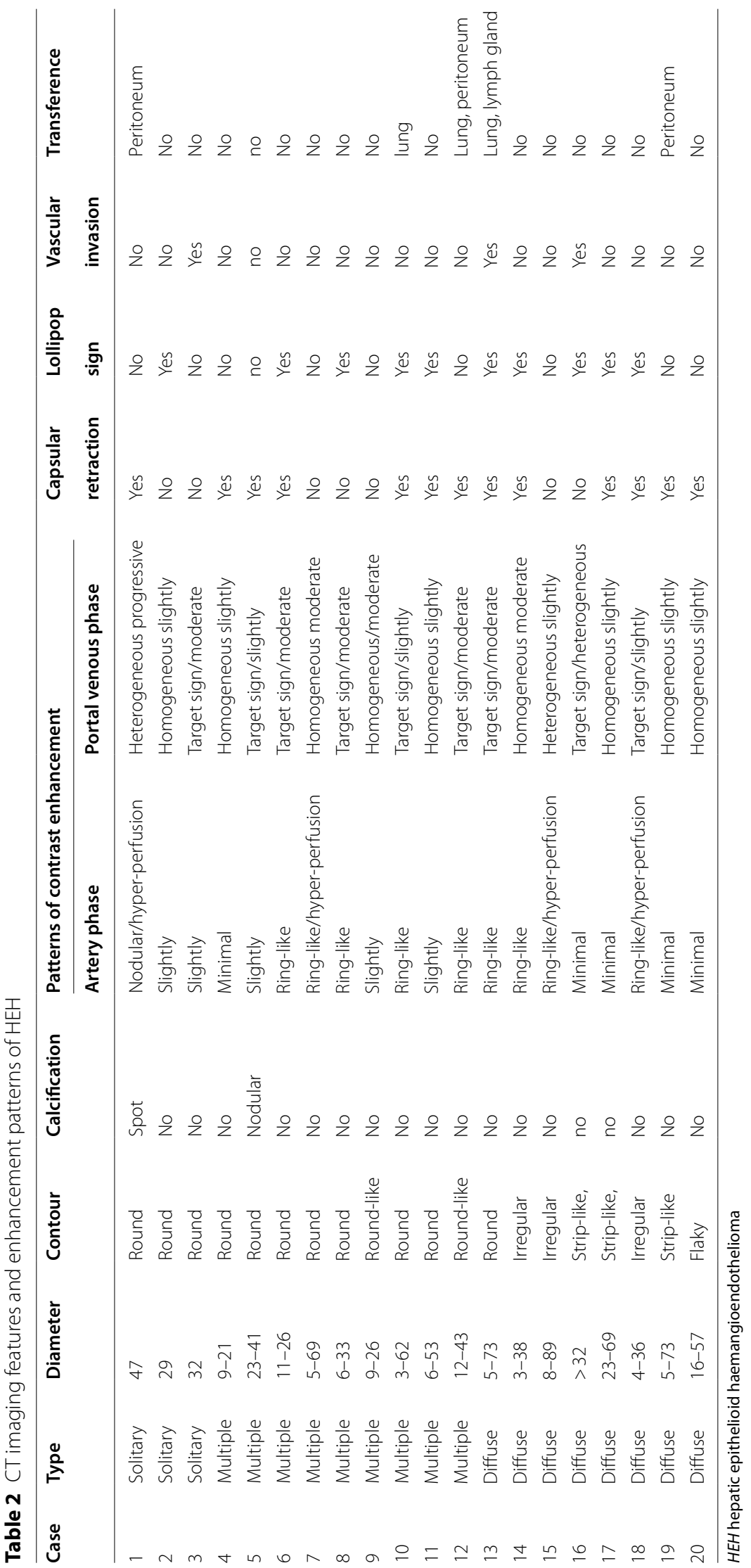




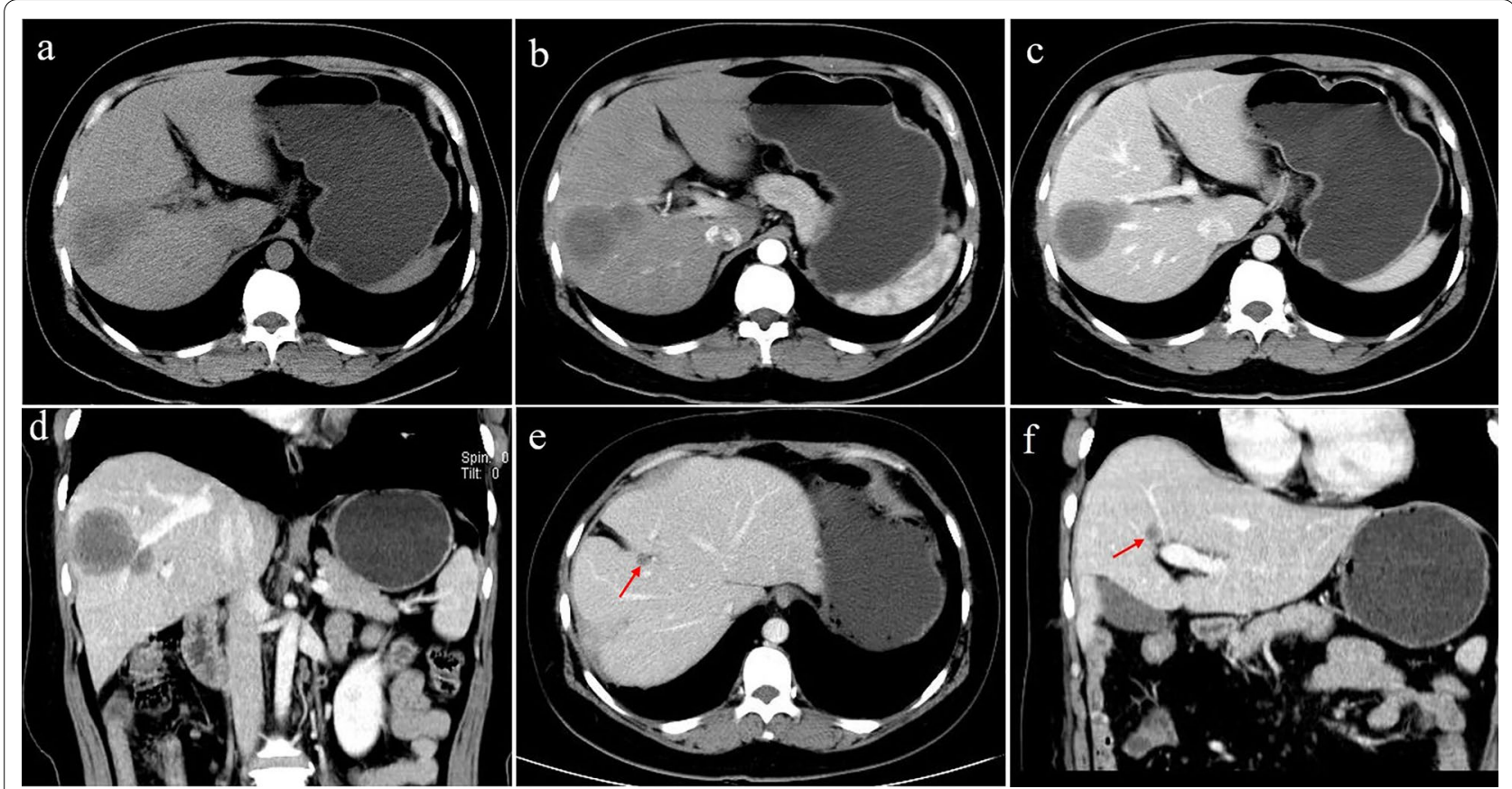

Fig. 2 A 41-year-old female with solitary nodular type HEH. An axial unenhanced CT image a shows a round nodule with ill-defined, heterogeneous hypodensity. An axial arterial phase image (b) shows rink-like enhancement. An axial portal phase image (c) shows "target" sign enhancement. Coronal reconstruction of the portal venous phase (d) shows a narrowing portal vein branch adjacent to the lesion. This patient was initially misdiagnosed with hepatic abscesses. On follow-up CT performed 10 months after resection $(\mathbf{e}, \mathbf{f})$, a new lesion was detected in the right lobe of the liver (red arrowheads)

detected in fewer patients with HEH than in those with LM $(p<0.05)$. All interobserver variability values were acceptable ( $\mathrm{K}$ coefficient range, $0.602-0.958$ ).

HEH masses had a significantly lower AER and ATNR than LMs $(p<0.05)$. Conversely, no significant differences in PER and PTNR were found. Using ROC analysis, cutoff values for AER, PER, ATNR and PTNR were 0.36, $0.63,0.69$ and 0.51, respectively; the corresponding AUCs (95\% CI) were $0.74(0.62-0.86), 0.57(0.42-0.72), 0.73$ (0.59-0.87), and 0.63 (0.49-0.76), respectively (Fig. 7). The above continuous variables were transformed into categorical variables according to the cut-off values. Table 5 summarises the sensitivity, specificity, and OR of each significant variable.

\section{Discussion}

HEH appears to have a clinical course between that of benign haemangioma and angiosarcoma. The World Health Organization classifies $\mathrm{HEH}$ as a malignant tumour of vascular origin [1]. Approximately 500 cases have been reported to date. HEH predominantly occurs in young and middle-aged women; the average age of the patients is 42 years old, and the female-to-male ratio is 1.6-2.0:1. The ratios of age and sex in this study are basically consistent with those reported in the literature [17]. The aetiology of HEH remains unknown; however, it may be associated with oral contraceptive use, exposure to polyethylene, alcohol, trauma or viral hepatitis [18]. Clinical manifestations of HEH are non-specific but most likely include upper abdominal discomfort, abdominal pain, poor acceptance, weight loss, jaundice, fever, and nausea [19]. Some diffuse and bilobar forms may show complicated clinical presentations, such as Budd-Chiari syndrome, portal hypertension or liver failure [5]. Biochemical examinations are frequently normal. In our study, 6 patients $(6 / 20,30 \%)$ exhibited a slight increase in aminotransferase and tumour markers. In contrast, most patients with LMs have obvious clinical symptoms (primary tumour- and cachexia-related), abnormal liver function, increased tumour markers and TAP. There was overall a significant difference between the two groups. Thus, a diagnosis of HEH may be considered for young and middle-aged women and asymptomatic patients with negative laboratory tests. LM mainly affected middle-aged and elderly individuals, with no significant sex predilection, and laboratory examinations were mostly abnormal. 

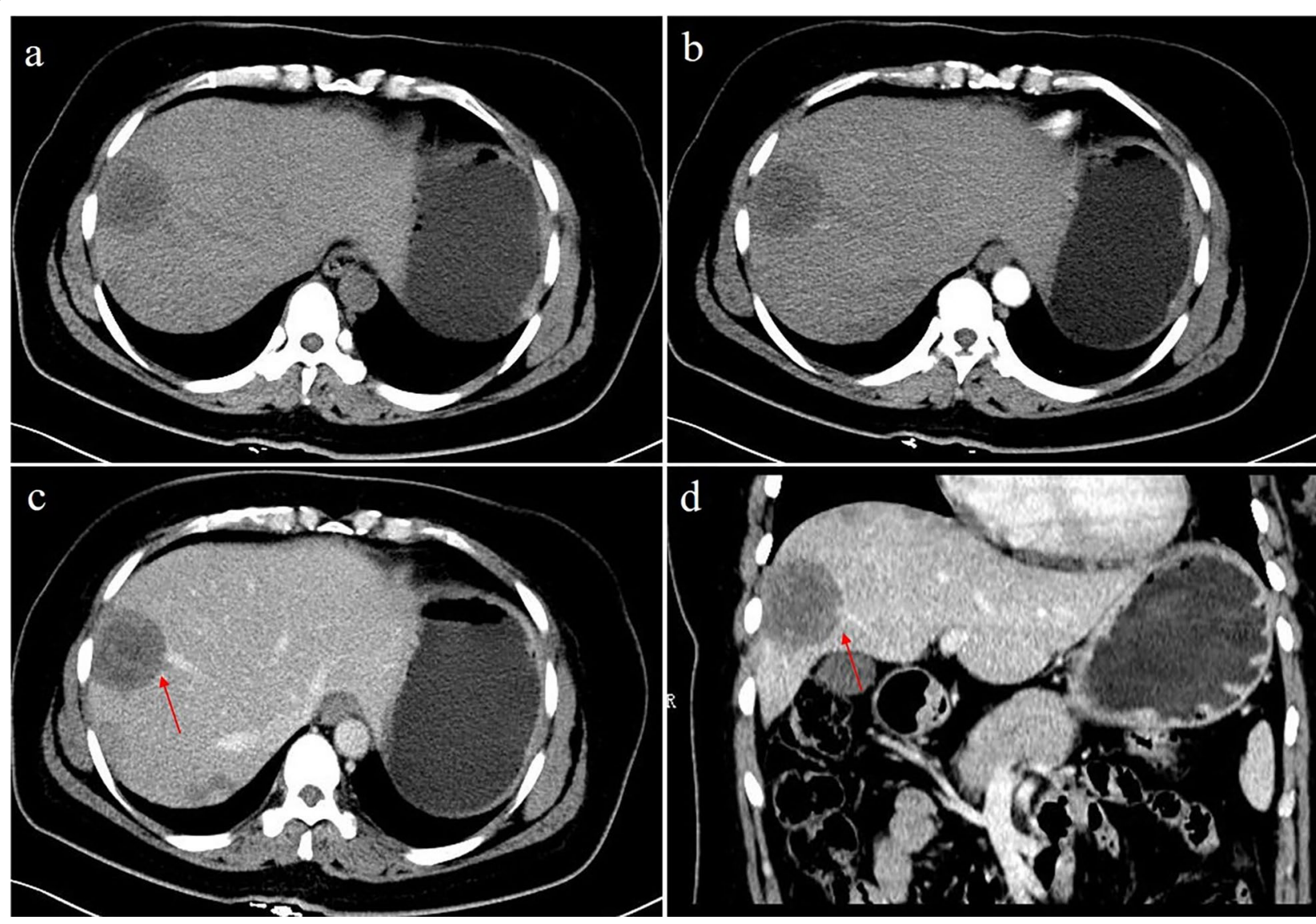

Fig. 3 A 48-year-old female with multifocal nodular type HEH. An axial unenhanced CT image (a) shows multiple round-like low-density lesions in the right lobe of the liver. The lesions show slight enhancement in the arterial phase (b). Axial and coronal reconstruction of portal phase CT images (c, d) show a larger nodule in the right lobe with portal veins entering and terminating in the periphery of the lesion (red arrow). This configuration resembles a typical "Iollipop" sign

CT imaging in our study also showed that the percentage of solitary nodular type cases was lower than that of the multifocal and diffuse types. This may be because the clinical symptoms of solitary nodule type $\mathrm{HEH}$ are not obvious; hence, it is difficult to detect. Certain studies have indicated that sub-marginal nodular lesions may be an early form of $\mathrm{HEH}$, as they later gradually transform into the diffuse type [20]. These three patterns may represent different stages of disease progression. In addition, most of the lesions displayed well-defined, round-like, low-density nodules, with a trend of distribution in perihepatic regions. Regarding diffuse type $\mathrm{HEH}$, there were often diffuse lesions of different sizes and ill-defined boundaries in the liver, and there was almost no normal liver parenchyma. These lesions often combine to form larger confluent masses. In our study, one patient with diffuse type $\mathrm{HEH}$ was misdiagnosed with hepatic fibrosis with a sub-marginal 'strip-like' sign accompanied by liver morphology abnormalities and proportion imbalance. Baron et al. reported these CT features [21, 22]. These signs are similar to liver cirrhosis, but there was no underlying liver disease in that case. Some diffuse type lesions in LM also showed strip-like enhancement, but there were no morphological changes in the liver and no obvious distribution characteristics. In 13 patients with HEH $(13 / 20,65 \%)$, retraction or flattening of the underlying liver capsule was observed. This percentage was higher than that reported by Zhao et al. [23], who found capsular retraction in 59.5\% of $\mathrm{HEH}$ lesions in Chinese patients. Hepatic capsule retraction may be due to fibrous contraction in the central region of the lesion. In fact, approximately 2 to $2.8 \%$ of liver tumours may show this sign, including LM, intrahepatic cholangiocarcinoma, and fibrolamellar hepatocellular carcinoma, among others [24]. Some LMs after systemic chemotherapy or the primary tumours themselves, such as colon cancer, breast cancer, and carcinoid tumours, contain fibroses, displaying capsule retraction. In this study, the number of patients with 

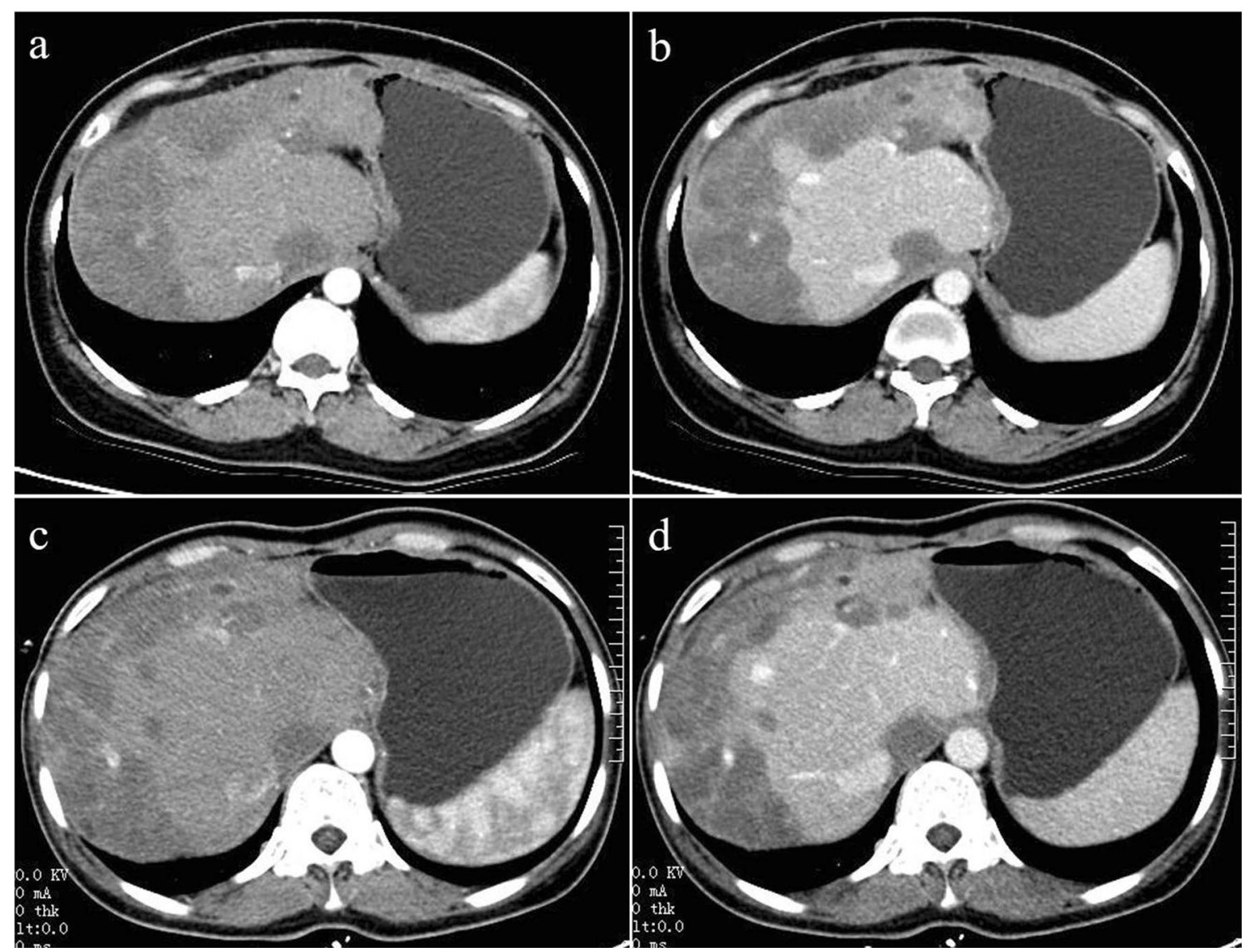

Fig. 4 A 40-year-old female with diffuse nodular type HEH. Axial arterial and portal phase CT images $(\mathbf{a}$, $\mathbf{b})$ show multiple, diffuse nodules that lack obvious enhancement and confluent masses with strip-like enhancement located at the subcapsular region of the liver, accompanied by liver morphological changes and proportion imbalance. On follow-up CT performed 18 months later without any treatment, axial arterial and portal phase images (c, d) show no obvious changes in the lesions

hepatic capsule retraction in the LM group $(6 / 60,10 \%)$ was significantly lower than that in the HEH group. In addition, peripheral cholangiocarcinoma may cause capsular retraction due to an abundant fibrous stroma and chronic peripheral biliary obstruction. At present, these findings still need to be combined with other signs, including peripheral biliary dilatation, delayed diffuse heterogeneous enhancement, and a low probability of the multifocal type. Two HEH patients $(2 / 20$, $10 \%)$ showed small spots and nodule-like calcifications. This was lower than in previous reports, in which $15 \%$ $25 \%$ of patients had intralesional calcifications [25, 26]. However, some patients with LM from colon cancer also had calcification. Therefore, intralesional calcification is another important but nonspecific feature that contributes to the diagnosis of HEH.

Based on dynamic enhancement scanning, 10 patients $(10 / 20,50 \%)$ had minimal to slightly homogeneous enhancement and $9(9 / 20,45 \%)$ mainly thin rinklike enhancement in the arterial phase. There was no significant difference compared with LM. Interestingly, lesions with ring-like and mild enhancement can exist in the same patient. In the portal phase, most lesions exhibited slight to moderate, gradually homogeneous enhancement, with some large lesions (diameter larger than $3 \mathrm{~cm}$ ) having heterogeneous enhancement. This is similar to the reports of Zhou et al. [27] and Klinger et al. [28]; indeed, this kind of enhancement characteristic in HEH is often misdiagnosed as LM. However, in LM, the rings are mostly thicker walled, more poorly defined, and obviously heterogeneous. We also used quantitative indicators (ER, TNR) to analyse enhancement characteristics, possibly reducing the influence of machine differences and individual variation. The results confirmed that HEH is a tumour with a poor blood supply, with an enhancement degree in the arterial phase lower than that in the adjacent liver parenchyma. The AER and ATNR of the HEH group were lower than those of the LM group $(p<0.05)$. Although the AUC of the AER was similar to that of the ATNR, the latter had higher sensitivity and 

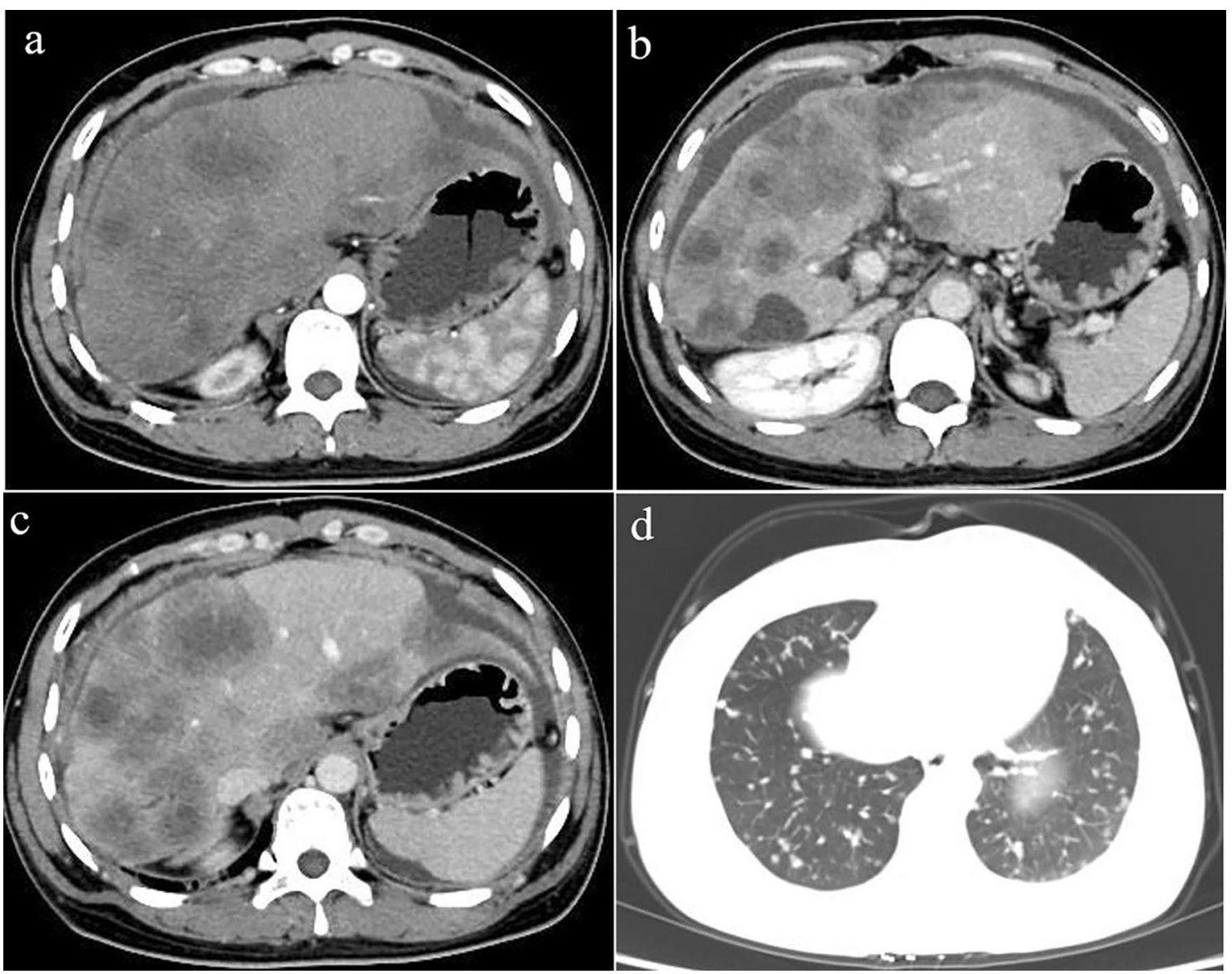

Fig. 5 A 34-year-old female with diffuse nodular type HEH. Axial and multiple phase contrast-enhanced CT images (a-c) show multiple, different-sized, coalescing, homogeneous, hypoattenuating nodules diffusing throughout the liver parenchyma. Masses show ring-like enhancement and the "target"sign. At the same time, there was arc-shaped effusion around the liver and peritoneal thickening. Peripheral distribution of masses and capsular retraction are also observed. The axial lung window image (d) shows lung involvement consisting of multiple round-like nodules

specificity. Therefore, the ATNR was more valuable for differential diagnosis of LM from HEH. These differences in the above enhancement characteristics and ratios are usually based on individual histopathologic characteristics. Peripheral proliferation of HEH remains active, and a large number of arterial-venous shunts are formed. Tumour cells and stroma are present in variable proportions, and the central stromal portion of the lesion can vary from myxoid to densely fibrotic, which may account for the slight and thin rim-like enhancement in the arterial phase and hypo-enhancement in the portal phase. In LM, the blood supply of the tumour cells around the lesion is abundant, and the centre of the lesion is usually accompanied by haemorrhage and necrosis; thus, the range of ring-like enhancement is often larger and the degree of enhancement more obvious than that of HEH.

In addition, the typical imaging findings of some lesions in HEH, such as the "target" sign and "lollipop" sign, were seen in the portal phase. Mamone et al. [12] reported that the "target" sign is generated by central hypocellular, loose, fibrous myxoid stroma and necrotic tissue and is surrounded by viable, hypercellular, peripheral proliferating tumour cells. The outer narrow hypodense rim corresponds to the peripheral avascular zone caused by vascular infiltration or occlusion of hepatic sinusoids and small vessels. As the tumour grows, the central stroma gradually degenerates and becomes sclerotic as the blood supply decreases. In our study, a few LM lesions showed a "target" sign. We speculate that the centre of the tumour would bleed and necrose as the legion enlarges and show heterogeneous enhancement similar to the "target" sign when the inner edge is smooth. The solitary "target" sign-enhanced lesion needs to be distinguished from an abscess; the central density of the abscess is lower because of liquefaction and necrosis, though 

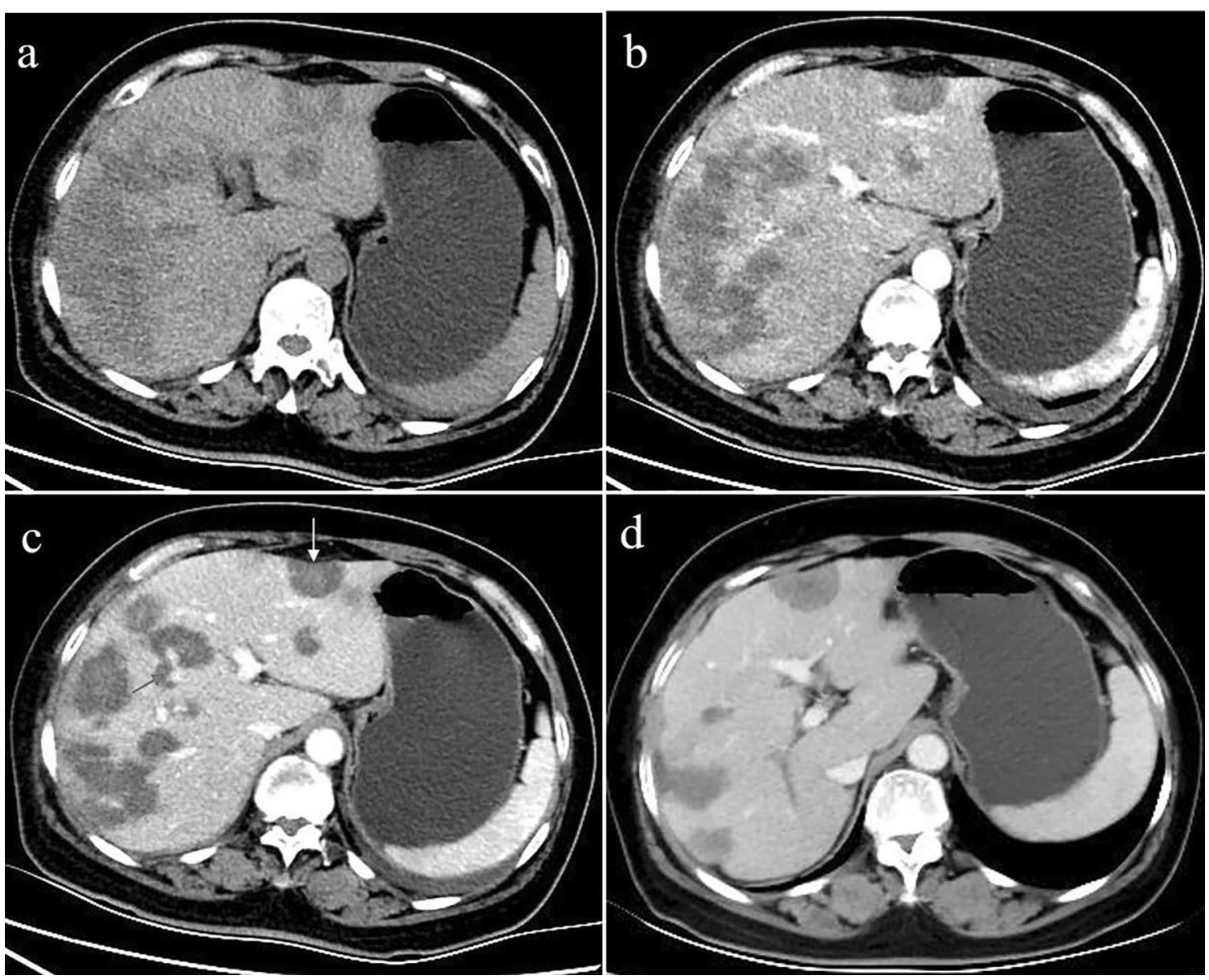

Fig. 6 A 52-year-old female with diffuse nodular type HEH. Axial unenhanced CT images (a) show diffuse coalescing hypoattenuating lesions. Arterial phase CT images (b) show slight enhancement, and some nodules confluent to strip-like or snowmelt are located subcapsularly in the liver. In the portal phase (c), the lesions are not progressively enhanced, a small branch of the portal vein is seen passing through the middle of the lesions (red arrow), and capsular retraction (yellow arrow). On follow-up CT performed 10 months after radiofrequency ablation, axial portal vein phase CT image (d) shows lesion reduction

the edge of the lesion shows more obvious enhancement due to the proliferation of inflammatory cells, and abnormal perfusion often appears around the arterial phase. In general, high fever, chills and increased leucocyte counts may be helpful in diagnosing abscesses. Another typical feature of HEH, the "lollipop" sign was first reported by Alomari [29]. This sign presents as a well-defined peripherally enhancing (or non-enhancing) tumour mass with an avascular core on enhanced images (the "candy" in the "lollipop") and the adjacent occluded vein (the stick) because HEH has a tendency to spread within the portal and hepatic vein branches. The vein should terminate smoothly at the edge or just within the rim of the lesion; vessels that traverse the entire lesion or are displaced, and collateral veins cannot be included in the sign. LM often directly invades adjacent blood vessels, resulting in wall stiffness, lumen stenosis or embolus formation. These two signs are conducive to distinguishing HEH from LM. Our study further confirmed the basic morphological features and typical signs of HEH described in the literature, but no other new signs were found.

Furthermore, HEH is often associated with extrahepatic organ invasion. In the study by Mehrabi et al., $36.6 \%$ of $\mathrm{HEH}$ patients had extrahepatic involvement, with organs such as the lung (8.5\%), local lymph nodes (7.7\%), peritoneum $(6.1 \%)$, bone $(4.9 \%)$, spleen $(3.2 \%)$ and diaphragm $(1.6 \%)$ as the most commonly involved sites [30]. Multipart CT scanning can be performed in one session to provide more information for an accurate diagnosis and comprehensive evaluation. In our study, 5 patients (25\%) with HEH showed extrahepatic involvement, fewer than patients with LM. Regardless, it is difficult to differentiate the imaging manifestations of extrahepatic organ invasion. 
Table 3 Comparison of clinical characteristics between $\mathrm{HEH}$ and LM

\begin{tabular}{|c|c|c|c|}
\hline Clinical characteristics & HEH $(n=20)$ & $\operatorname{LM}(n=60)$ & $P$ value \\
\hline Age $(\text { year) })^{\mathrm{a}}$ & $42.95 \pm 12.29$ & $57.40 \pm 9.50$ & 0.000 \\
\hline $\operatorname{Sex}^{*}$ & & & 0.007 \\
\hline Male & $5(25)$ & $36(60)$ & \\
\hline Female & $15(75)$ & $24(40)$ & \\
\hline \multicolumn{4}{|l|}{ Main symptoms } \\
\hline Abdominal discomfort/pain* & $6(30)$ & $37(62)$ & 0.014 \\
\hline Fever* & $1(5)$ & $3(5)$ & 1.000 \\
\hline Weak* & $1(5)$ & $12(20)$ & 0.221 \\
\hline Weight loss* & $1(5)$ & $42(70)$ & 0.000 \\
\hline Poor appetite* & $1(5)$ & $25(42)$ & 0.002 \\
\hline Melena/hematemesis* & $0(0)$ & $27(45)$ & 0.000 \\
\hline Asymptomatic* & $10(50)$ & $8(13)$ & 0.002 \\
\hline Liver function abnormal* & $6(30)$ & $34(57)$ & 0.039 \\
\hline Tumour mark positive* & $6(30)$ & $28(47)$ & 0.035 \\
\hline CA 199 & $1(5)$ & $38(63)$ & \\
\hline CA125 & $4(20)$ & $39(65)$ & \\
\hline CEA & $1(5)$ & $41(68)$ & \\
\hline Tumour abnormal protein rise* & $1(5)$ & $49(82)$ & 0.000 \\
\hline
\end{tabular}

HEH hepatic epithelioid haemangioendothelioma, $L M$ liver metastases

* Data are number of patients with the percentage in parenthesis; $p$ values were calculated from $x^{2}$ test and Fisher' exact test

${ }^{a}$ Data are mean \pm standard deviation; $p$ values were calculated from student $t$ test

The clinical prognosis of $\mathrm{HEH}$ is variable and unpredictable, ranging from an aggressive and fulminant course to possible long-term survival without definitive therapy [31]. The usual treatment for HEH includes liver resection, transcatheter arterial chemoembolisation, chemotherapy, radiation therapy and liver transplantation. Systemic treatment options include anti-angiogenic drugs such as pazopanib that inhibit $\mathrm{HEH}$ growth [32], immunotherapy with interferon $\alpha-2 B$, kinase inhibitors, and chemotherapy [33]. The prognosis of $\mathrm{HEH}$ is closely related to the size and number of lesions, the biological behaviour of the tumour (cellularity and cell necrosis), the degree of multiple organ damage, and the choice of treatment [10, 34]. According to our results, "target" sign has a moderate correlation with treatment results. Among 9 patients with the "target" sign of HEH, 8 cases were multiple and diffuse types; 6 cases progressed after treatment, which further indicates that tumour cells in the "target" sign lesion proliferate actively, with a poor prognosis. However, the overall survival rate of HEH patients was significantly higher than that of LM patients. Surgical resection is the best choice for the solitary nodular type with lesions confined to one liver lobe. Additionally, TACE is recommended if lesions are unresectable. Comprehensive treatment is more appropriate for $\mathrm{HEH}$ with multiple and diffuse types, though local resection easily leads to recurrence. In our study, 3 patients $(3 / 4,75 \%)$ showed a new lesion after resection, which is higher than in previous reports. This may be related to the pathological features of the lesion itself and the duration of follow-up. During follow-up, among the 3 patients who received interventional therapy, 2 had reduced lesions, and 1 showed no obvious change. Our findings also indicate that $\mathrm{HEH}$ is a low- to moderate malignant tumour and that proliferation of new tumour cells is slow after focal ischaemic necrosis. Regardless of extrahepatic involvement, liver transplantation is the most effective treatment for unresectable HEH [35]. In this study, no patients underwent liver transplantation, which may be related to their financial situation, understanding of the disease and other factors.

Our study has several limitations. First, because the study was retrospective, there was inevitable selection bias, especially in the primary diseases of LM. Nonetheless, liver metastases from different organs have some similar imaging features. We chose more primary tumour types according to the incidence of LM instead of certain tumour with equal numbers. Second, we set the ROI as the whole tumour rather than its periphery because most HEH lesions showed homogeneous minimal and slight enhancement, and it was difficult to define the active part around the tumour for some lesions with heterogeneous enhancement. However, whether the comparison of tumour periphery is meaningful for distinguishing $\mathrm{HEH}$ from LM needs further study. Third, most patients had multiple lesions with an average diameter of $3.85 \mathrm{~cm}$, but we only selected 3 of them. To minimise selection bias, we limited the lesion diameter to $2-5 \mathrm{~cm}$. Fourth, we used different types of CT scanners and protocols over the 8 years of the study. Nevertheless, we believe that morphological features may be unaffected by these nonconformities, and the application of ER and TNR may have greatly reduced their influence. Fifth, patients with different follow-up times had different prognoses. Larger studies are required to identify new signs associated with $\mathrm{HEH}$ and to confirm the present findings.

\section{Conclusions}

In conclusion, dynamic $\mathrm{CT}$ imaging revealed special features of $\mathrm{HEH}$, including subcapsular location and nodule coalescence, capsular retraction, intralesional calcifications, and slight and peripheral ring-like enhancement. When the typical "target" sign and "lollipop" sign are present, HEH should be highly suspected. CT imaging and a lower AER and TNR can, when combined with clinical data, help to improve differential diagnosis of HEH. Additionally, scanning multiple sites is conducive to accurate clinical grading and prognosis evaluation. 
Table 4 Comparison of quantitative and qualitative CT findings between $\mathrm{HEH}$ and LM

\begin{tabular}{|c|c|c|c|c|}
\hline CT findings & HEH $(n=20)$ & $\operatorname{LM}(n=60)$ & $P$ value & $k$ coefficient \\
\hline Classification* & & & 0.963 & 0.912 \\
\hline Solitary nodular type & $3(15)$ & $8(13)$ & & \\
\hline Multifocal nodular type & $9(45)$ & $29(49)$ & & \\
\hline Diffuse types & $8(40)$ & $23(38)$ & & \\
\hline Location* & & & 0.000 & 0.897 \\
\hline Scattered distribution & $7(35)$ & $51(85)$ & & \\
\hline Liver subcapsular & $13(65)$ & $9(15)$ & & \\
\hline Contour* & & & 0.138 & 0.846 \\
\hline Round/round-like & $13(65)$ & $30(50)$ & & \\
\hline Irregular & $3(15)$ & $24(40)$ & & \\
\hline Strip-like/flaky & $4(20)$ & $6(10)$ & & \\
\hline Border* & & & 0.005 & 0.851 \\
\hline Well defined & $13(65)$ & $18(30)$ & & \\
\hline III defined & $7(35)$ & $42(70)$ & & \\
\hline Density of plain scan* & & & 0.639 & 0.803 \\
\hline Low & $17(85)$ & $45(75)$ & & \\
\hline Equal & $1(5)$ & $6(10)$ & & \\
\hline High & $2(10)$ & $9(15)$ & & \\
\hline Enhancement patter and degree in $\mathrm{AP}^{*}$ & & & 0.005 & 0.768 \\
\hline Minimal & $5(25)$ & $3(5)$ & & \\
\hline Slightly & $5(25)$ & $13(22)$ & & \\
\hline Moderate/obvious & $0(0)$ & $17(28)$ & & \\
\hline Ring-like & $9(45)$ & $27(45)$ & & \\
\hline Nodular & $1(5)$ & $0(0)$ & & \\
\hline Enhancement patter and degree in PVP* & & & 0.068 & 0.755 \\
\hline Slightly & $12(60)$ & $22(37)$ & & \\
\hline Moderate & $8(40)$ & $38(63)$ & & \\
\hline \multicolumn{5}{|l|}{ Special signs } \\
\hline Capsular retraction* & $13(65)$ & $6(10)$ & 0.000 & 0.892 \\
\hline Target sign* & $9(45)$ & $7(12)$ & 0.004 & 0.794 \\
\hline Lollipop sign* & $10(50)$ & $3(5)$ & 0.000 & 0.813 \\
\hline Vascular invasion* & $3(15)$ & $12(20)$ & 0.869 & 0.773 \\
\hline Calcification* & $2(10)$ & $4(7)$ & 0.493 & 0.958 \\
\hline \multicolumn{5}{|l|}{ Extrahepatic involvement } \\
\hline Lung* & $3(15)$ & $16(27)$ & 0.448 & 0.742 \\
\hline Peritoneum* & $3(15)$ & $26(43)$ & 0.022 & 0.679 \\
\hline Lymph node* & $1(5)$ & $37(62)$ & 0.000 & 0.703 \\
\hline Bone* & $0(0)$ & $7(12)$ & 0.026 & 0.602 \\
\hline$A E R^{a}$ & $0.26 \pm 0.10$ & $0.37 \pm 0.13$ & 0.001 & \\
\hline$P E R^{\mathrm{a}}$ & $0.58 \pm 0.13$ & $0.61 \pm 0.15$ & 0.369 & \\
\hline ATNR $^{a}$ & $0.69 \pm 0.05$ & $0.71 \pm 0.04$ & 0.022 & \\
\hline PTNR $^{a}$ & $0.51 \pm 0.04$ & $0.53 \pm 0.05$ & 0.056 & \\
\hline
\end{tabular}

HEH hepatic epithelioid haemangioendothelioma, LM liver metastases, AER arterial enhancement ratio, PER portal enhancement ratio, ATNR arterial tumour to normal parenchyma ratio, PTNR portal tumour to normal parenchyma ratio

* Data are number of patients with the percentage in parenthesis; $p$ values were calculated from $x^{2}$ test and Fisher' exact test

${ }^{a}$ Data are mean \pm standard deviation; $p$ values were calculated from student $t$ test 


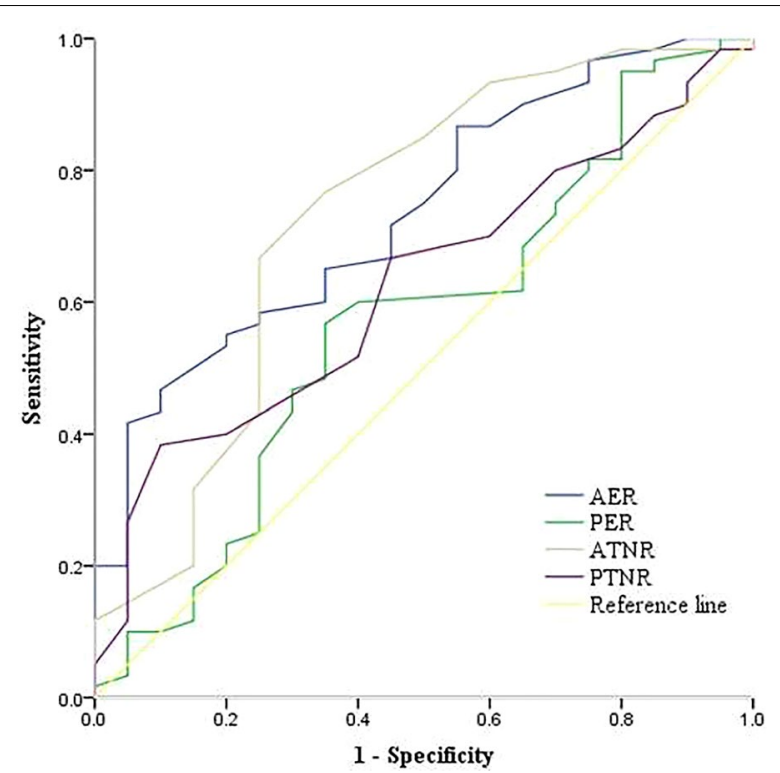

Fig. 7 ROC curves for the arterial enhancement ratio (AER), portal enhancement ratio (PER), arterial tumour to normal parenchyma ratio (ATNR), and portal tumour to normal parenchyma ratio (PTNR). There were significant differences between the arterial enhancement ratio and the arterial tumour to the normal parenchyma

Table 5 Sensitivity and specificity of CT finding in diagnosis of $\mathrm{HEH}$

\begin{tabular}{llll}
\hline CT findings & Sensitivity (\%) & Specificity & OR (95\%Cl) \\
\hline Liver subcapsular & 65.0 & 85.0 & $10.52(3.30-33.58)$ \\
Capsular retraction & 65.0 & 90.1 & $16.71(4.80-58.18)$ \\
Target sign & 45.0 & 88.3 & $6.2(1.90-20.20)$ \\
Lollipop sign & 50.0 & 96.7 & $14.0(3.67-53.50)$ \\
AER $<0.36$ & 80.0 & 48.3 & $3.74(1.12-12.51)$ \\
ATNR $<0.69$ & 75.0 & 78.3 & $78.3(3.32-35.44)$ \\
\hline
\end{tabular}

HEH hepatic epithelioid haemangioendothelioma, AER arterial enhancement ratio, ATNR arterial tumour to normal parenchyma ratio

\begin{abstract}
Abbreviations
AER: Arterial enhancement ratio; ATNR: Arterial tumour to normal parenchyma ratio; AUC: Areas under the curve; Cls: Confidence intervals; ER: Enhancement ratio; $\mathrm{HEH}$ : Hepatic epithelioid haemangioendothelioma; $\mathrm{HU}$ : Hounsfield units; LM: Liver metastasis; OR: Odds ratio; PER: Portal venous enhancement ratio; PTNR: Portal tumour to normal parenchyma ratio; RFA: Radio frequency ablation; ROC: Receiver operating characteristic; ROI: Regions of interest; TACE: Transcatheter arterial chemoembolisation; TNR: Ratio of tumour to normal parenchyma.
\end{abstract}

\section{Acknowledgements}

The authors would like to thank numerous individuals participated in this study and and Professor Jianbo Gao for critically reviewing the manuscript.

\section{Authors' contributions}

XPW: manuscript preparation and submission, literature research, data analysis, statistical analysis, manuscript editing and review; PL: data acquisition and literature research; PJL: literature research and data analysis; RL: data acquisition; PH: data acquisition; JBG: study conception and design, manuscript review and guarantor of integrity of the entire study. All authors read and approved the final manuscript.

\section{Funding}

This research did not receive any specific grant from funding agencies in the public, commercial, or not-for-profit sectors.

\section{Availability of data and materials}

The datasets used and/or analysed during the current study are available from the corresponding author on reasonable request.

\section{Declarations}

\section{Ethics approval and consent to participate}

This retrospective study was approved by the Institutional Review Board of the First Affiliated Hospital of Zhengzhou University, and the requirement for informed consent was waived.

\section{Consent for publication}

Consent to publication has been signed by each participant.

\section{Competing interests}

The authors declare that they have no competing interests.

Received: 11 August 2021 Accepted: 14 December 2021

Published online: 20 January 2022

\section{References}

1. Cardinal J, de Vera ME, Marsh JW et al (2009) Treatment of hepatic epithelioid hemangioendothelioma: a single-institution experience with 25 cases. Arch Surg 144(11):1035-1039

2. Amin S, Chung H, Jha R (2011) Hepatic epithelioid hemangioendothelioma: MR imaging findings. Abdom Imaging 36(4):407-414

3. Weiss SW, Enzinger FM (1982) Epithelioid hemangioendothelioma a vascular tumor often mistaken for a carcinoma. Cancer 50(5):970-981

4. Ishak KG, Sesterhenn IA, Goodman MZ, Rabin L, Stromeyer FW (1984) Epithelioid hemangioendothelioma of the liver: a clinicopathologic and follow-up study of 32 cases. Hum Pathol 15(9):839-852

5. Elleuch N, Dahmani W, Aida Ben S et al (2018) Hepatic epithelioid hemangioendothelioma: a misdiagnosed rare liver tumor. Presse Med 47(2):182-185

6. Kubo N, Harimoto N, Araki K et al (2018) The feature of solitary small nodular type of hepatic epithelioid hemangioendothelioma. Case Rep Gastroenterol 12(2):402-410

7. Dong Y, Wang WP, Cantisani V et al (2016) Contrast-enhanced ultrasound of histologically proven hepatic epithelioid hemangioendothelioma. World J Gastroenterol 22(19):4741-4749

8. Gan LU, Chang R, Jin H, Yang L (2016) Typical CT and MRI signs of hepatic epithelioid hemangioendothelioma. Oncol Lett 11(3):1699-1706

9. Kim EH, Rha SE, Lee YJ, Yoo leR, Jung ES, Byun JY (2015) CT and MR imaging findings of hepatic epithelioid hemangioendotheliomas: emphasis on single nodular type. Abdom Imaging 40(3):500-509

10. Wang W, Liu G, Hu P et al (2020) Imaging characteristics and prognostic values of hepatic epithelioid hemangioendothelioma on ${ }^{18} \mathrm{~F}-\mathrm{FDG}$ PET/CT. Clin Exp Med 20(4):557-567

11. Nakai H, Arizono S, Isoda H, Togashi K(2019) Imaging characteristics of liver metastases overlooked at contrast-enhanced CT. AJR Am J Roentgenol 212(4):782-787

12. Mamone G, Miraglia R (2019) The "'Target sign"' and the "'Lollipop sign"' in hepatic epithelioid hemangioendothelioma. Abdom Radiol (NY) 44(4):1617-1620

13. Park HJ, Kim HJ, Kim KW et al (2020) Comparison between neuroendocrine carcinomas and well-differentiated neuroendocrine tumors of the pancreas using dynamic enhanced CT. Eur Radiol 30(9):4772-4782 
14. Lv P, Liu J, Chai Y, Yan X, Gao J, Dong J (2017) Automatic spectral imaging protocol selection and iterative reconstruction in abdominal CT with reduced contrast agent dose: initial experience. Eur Radiol 27(1):374-383

15. Li X, Yi W, Zeng Q (2018) CT features and differential diagnosis of primary pulmonary mucoepidermoid carcinoma and pulmonary adenoid cystic carcinoma. J Thorac Dis 10(12):6501-6508

16. Hu HJ, Jin YW, Jing QY, Shrestha A, Cheng NS, Li FY (2016) Hepatic epithelioid hemangioendothelioma: dilemma and challenges in the preoperative diagnosis. World J Gastroenterol 22(41):9247-9250

17. Sanduzzi-Zamparelli M, Rimola J, Montironi C et al (2020) Hepatic epithelioid hemangioendothelioma: an international multicenter study. Dig liver Dis 52(9):1041-1046

18. Kou K, Chen YG, Zhou JP et al (2020) Hepatic epithelioid hemangioendothelioma: update on diagnosis and therapy. World J Clin Cases 8(18):3978-3987

19. Studer LL, Selby DM (2018) Hepatic epithelioid hemangioendothelioma. Arch Pathol Lab Med 142(2):263-267

20. Giardino A, Miller FH, Kalb B et al (2016) Hepatic epithelioid hemangioendothelioma: report from three university centers. Radiol Bras 49(5):288-294

21. Baron PW, Amankonah T, Cubas RF et al (2014) Diffuse hepatic epithelioid hemangioendothelioma developed in a patient with hepatitis C cirrhosis. Case Rep Transplant 2014:694903

22. Ehman EC, Torbenson MS, Wells ML et al (2018) Hepatic tumors of vascular origin: imaging appearances. Abdom Radiol (NY) 43(8):1978-1990

23. Zhao XY, Rakhda MI, Habib S et al (2014) Hepatic epithelioid hemangioendothelioma: a comparison of Western and Chinese methods with respect to diagnosis, treatment and outcome. Oncol Lett 7(4):977-983

24. Din NU, Rahim S, Asghari T, Abdul-Ghafar J, Ahmad Z (2020) Hepatic epithelioid hemangioendothelioma: case series of a rare vascular tumor mimicking metastases. Diagn Pathol 15(1):120

25. Ganeshan D, Pickhardt PJ, Morani AC et al (2020) Hepatic hemangioendothelioma: CT, MR, and FDG-PET-CT in 67 patients-a bi-institutional comprehensive cancer center review. Eur Radiol 30(5):2435-2442

26. Bruegel M, Muenzel D, Waldt S, Specht K, Rummeny EJ (2011) Hepatic epithelioid hemangioendothelioma: findings at CT and MRI including preliminary observations at diffusion-weighted echo-planar imaging. Abdom Imaging 36(4):415-424

27. Zhou L, Cui MY, Xiong J et al (2015) Spectrum of appearances on CT and MRI of hepatic epithelioid hemangioendothelioma. BMC Gastroenterol 15:69

28. Klinger C, Stuckmann G, Dietrich CF et al (2019) Contrast-enhanced imaging in hepatic epithelioid hemangioendothelioma: retrospective study of 10 patients. Z Gastroenterol 57(6):753-766

29. Alomari Al (2006) The lollipop sign: a new cross-sectional sign of hepatic epithelioid hemangioendothelioma. Eur J Radiol 59(3):460-464

30. Mehrabi A, Kashfi A, Fonouni H et al (2006) Primary malignant hepatic epithelioid hemangioendothelioma: a comprehensive review of the literature with emphasis on the surgical therapy. Cancer 107(9):2108-2121

31. Onishi Y, Kusumoto M, Motoi N et al (2021) Natural history of epithelioid hemangioendothelioma of the liver: CT findings of 15 cases. Acad Radiol 28(6):778-782

32. Mistry AM, Gorden DL, Busler JF, Coogan AC, Kelly BS (2012) Diagnostic and therapeutic challenges in hepatic epithelioid hemangioendothelioma. J Gastrointest Cancer 43(4):521-525

33. Rosenberg A, Agulnik M (2018) Epithelioid hemangioendothelioma: update on diagnosis and treatment. Curr Treat Options Oncol 19(4):19

34. Mehrabi A, Hoffmann K, Weiss KH et al (2016) Long term follow up after resectionemphasizes the role of surgery in primary hepatic epithelioid hemangioendothelioma. Ann Med Surg (Lond) 11:1-4

35. Noh OK, Kim SS, Yang MJ et al (2020) Treatment and prognosis of hepatic epithelioid hemangioendothelioma based on SEER data analysis from 1973 to 2014. Hepatobiliary Pancreat Dis Int 19(1):29-35

\section{Publisher's Note}

Springer Nature remains neutral with regard to jurisdictional claims in published maps and institutional affiliations.

\section{Submit your manuscript to a SpringerOpen ${ }^{\circ}$ journal and benefit from:}

- Convenient online submission

- Rigorous peer review

- Open access: articles freely available online

- High visibility within the field

- Retaining the copyright to your article

Submit your next manuscript at $\boldsymbol{\Delta}$ springeropen.com 